TRANSACTIONS OF THE

AMERICAN MATHEMATICAL SOCIETY

Volume 363, Number 5, May 2011, Pages 2701-2714

S 0002-9947(2010)05211-0

Article electronically published on December 10, 2010

\title{
GEOMETRICALLY FINITE AND SEMI-RATIONAL BRANCHED COVERINGS OF THE TWO-SPHERE
}

\author{
GUIZHEN CUI AND YUNPING JIANG
}

\begin{abstract}
In 1982, Thurston gave a necessary and sufficient condition for a critically finite branched covering of the two-sphere to itself to be combinatorially equivalent to a rational map. We discuss extending this result to geometrically finite rational maps. We give an example to show that Thurston's original condition is not sufficient. This example is topologically pathological near accumulation points of the postcritical set. We give two conditions forbidding such pathology, show that they are equivalent, and (in a sequel to the present paper) will show that Thurston's condition together with this tameness is both necessary and sufficient to characterize geometrically finite rational maps.
\end{abstract}

\section{INTRODUCTION}

The discovery of a combinatorial condition of a rational map among all branched coverings of the two-sphere is an interesting and important problem in complex dynamical systems. The simplest complex dynamical systems are the postcritically finite ones. In 1982, Thurston gave a necessary and sufficient combinatorial condition for a critically finite rational map among all critically finite branched coverings and stated a rigidity theorem. A complete proof of this theorem was written by Douady and Hubbard in 6] (see Theorem 2.1). This theorem is used to give combinatorial models for parameter spaces of rational maps (the model for the Mandelbrot set; M. Rees' models for slices of quadratic parameter space; models for cubic Newton's method parameter space, etc.) and is therefore important.

It is then natural to ask the following question: Is Thurston's combinatorial condition also necessary and sufficient for any rational maps among all branched coverings? McMullen proved in [9] that Thurston's condition is essentially necessary for all rational maps. The only possible trivial failure cases are inside Siegel disks or Herman rings (see Theorem 2.2). Therefore, the next question is the following: Excluding these trivial failure cases, is Thurston's combinatorial condition necessary and sufficient for any rational maps among all branched coverings? The next bestunderstood class of maps are the geometrically finite ones. Our purpose is to generalize Thurston's criteria to this setting. For quadratic polynomials, this has been done only very recently by Brown (a recent Ph.D. student of Hubbard) in his thesis [1. So, there has been only a tiny amount of progress on this topic until our work in this paper and in a sequel to the present paper [4, 12]. Our results

Received by the editors June 2, 2009 and, in revised form, September 18, 2009.

2010 Mathematics Subject Classification. Primary 37F20, 37F10, 30D05.

Key words and phrases. Geometrically finite branched covering, semi-rational branched covering, sub-hyperbolic semi-rational branched covering. 
in this paper show that a generalization of Thurston's criteria to the geometrically finite maps is highly non-trivial. Actually, we give a negative answer to the above question in the case of the geometrically finite maps in this paper (see Theorem 4.1).

To obtain this negative answer, we study the local combinatorial structure of a geometrically finite branched covering of the two-sphere. We first introduce a concept called locally combinatorially attracting (see Definition 3.2). We then prove that a geometrically finite branched covering of the two-sphere which is locally combinatorially attracting at a limiting point of its post-critical set is combinatorially equivalent to a branched covering which is holomorphic in a neighborhood of the corresponding limiting point of its post-critical set, and this corresponding limiting point is an attractive or super-attractive periodic point of this map (see the proof of Theorem 3.1). During the proof of Theorem 3.1, we introduce another concept called a combinatorially invariant shrinking family of nested curves (see Definition 3.4). We prove that the two concepts, locally combinatorially attracting and a combinatorially invariant shrinking family of nested curves, are essentially the same at a non-branched limiting point of its post-critical set (see Lemma 3.1). From the latter topologically tameness condition, we prove that a geometrically finite rational map is always locally combinatorially attracting at all limiting points of its post-critical set (see the proof of Theorem 3.1).

Finally, we construct an example of a geometrically finite branched covering of the two-sphere after completely understanding the above two concepts. Thurston's combinatorial condition is satisfied by this example, but it is not combinatorially equivalent to any rational map. Thus the necessity of Thurston's combinatorial condition fails by this example (see Theorem 4.1).

Due to this negative answer, we introduce semi-rational branched coverings and sub-hyperbolic semi-rational branched coverings (see Definition 3.1). We prove that a semi-rational branched covering is always combinatorially equivalent to a sub-hyperbolic semi-rational branched covering (see Theorem 3.1). We then introduce a combinatorial and locally holomorphical equivalence relation among all sub-hyperbolic semi-rational branched coverings (see Definition 5.11).

The next question is the following: Is Thurston's combinatorial condition necessary and sufficient for a geometrically finite rational map among all semi-rational branched coverings under the combinatorial equivalence relation? We know in this paper that a semi-rational branched covering is always combinatorially equivalent to a sub-hyperbolic semi-rational branched covering (see Theorem 3.1). So this question can be asked as follows: Is Thurston's combinatorial condition necessary and sufficient for a sub-hyperbolic geometrically finite rational map among all subhyperbolic semi-rational branched coverings under our combinatorial and locally holomorphical equivalence relation? Since a geometrically finite rational map has no Siegel disk and Herman ring, the necessary condition just follows McMullen's Theorem (see Theorem 2.2). However, the sufficient condition is a much harder and more interesting problem. We studied this topic in the paper [2, first circulated in 1994. The paper was then divided into two parts and published in a workshop proceedings as $[3,4$. The current paper is a rewritten version of [3] and is more concerned with setting up an appropriate way to answer the sufficiency condition. In the paper [4, we stated the following theorem:

Theorem 1.1. Suppose $f$ is a sub-hyperbolic semi-rational branched covering of the two-sphere. Then $f$ is combinatorially and locally holomorphically equivalent 
to a rational map $R$ if and only if $f$ has no Thurston obstructions. In this case, the rational map $R$ is unique up to conjugation by Möbius transformations of the Riemann sphere.

See Definition 5.1 for the concept of a combinatorial and locally holomorphical equivalence relation among all sub-hyperbolic semi-rational branched coverings of the two-sphere.

The proof of this theorem in [4, and also in its improved version [5], is quite involved. A combinatorially complex and expositionally formidable surgery argument is used to reduce the problem to that of Thurston's original postcritically finite setup (see Theorem 2.1), together with checking that certain gluing data are analytically realizable. In the paper [12, a new and simpler proof was given by completely giving up the idea in the paper [4]. This new proof adapted some arguments used in the proof in Douady and Hubbard's paper [6] directly and combined with an idea of constructing a shielding ring around every accumulation point in the limiting set of the post-critical set. Then we proved that the bounded geometry is valid due to these shielding rings. This new proof is easier and checkable and also gives some new interpretation of the proof of Thurston's Theorem in DouadyHubbard's paper [6. Thus a complete and understandable proof of Theorem 1.1 is available in [12. Combining Theorem 1.1] and Theorem 3.1, we eventually obtained a generalization of Thurston's criteria to the geometrically finite ones as follows:

Theorem 1.2. Suppose $f$ is a semi-rational branched covering of the two-sphere. Then $f$ is combinatorially equivalent to a rational map $R$ if and only if $f$ has no Thurston obstructions. However, the rational map $R$ is not unique up to conjugation by Möbius transformations of the Riemann sphere.

Remark 1.1. In the above theorem, the statement that the rational map $R$ is not unique up to conjugations by Möbius transformations of the Riemann sphere is itself an interesting topic. This result is a consequence of the study of the local combinatorial structure of a super-attractive, attractive, or parabolic periodic cycle for a holomorphic map (see the proof of Theorem 3.1 in $\S 3$ and the proof of Theorem 4.1 in $\S 4$ ). From this statement we have the following: First, combinatorial equivalence is insensitive to quasiconformal deformations. Secondly, combinatorial equivalence cannot distinguish between attractive and parabolic periodic cycles. Therefore, the new concept, combinatorial and locally holomorphical (CLH) equivalence relation, introduced in $\S 5$ becomes an interesting and important issue.

A combinatorial characterization of rational maps or entire functions is important and interesting in complex dynamical systems. Besides the work we mentioned above, we would also like to mention Hubbard, Schleicher, and Shishikura's paper 7 ] on the exponential family, and a thesis of Zhang (a recent Ph.D. student of Jiang) on simple Siegel disk type polynomials [11].

This article is arranged as follows. In $\S 2$, we review Thurston's Theorem and McMullen's Theorem and Mañé's Theorem. Then we define geometrically finite branched coverings which contain all geometrically finite rational maps (see Definition 2.1).

In $\S 3$, we define semi-rational and sub-hyperbolic semi-rational branched coverings (see Definition 3.1). We also define our locally combinatorially attracting condition (see Definition 3.2). We prove that a semi-rational branched covering is locally combinatorially attracting at any limiting point of its post-critical set (see 
the proof of Theorem 3.1). Therefore, a semi-rational branched covering is always combinatorially equivalent to a sub-hyperbolic semi-rational branched covering (see Theorem 3.1). We would like to note that the most interesting result in this study is that a geometrically finite rational map with parabolic cycles is combinatorially equivalent to a sub-hyperbolic rational map, which is a geometrically finite rational map without parabolic cycles

In $\S 4$, we apply a technique called the Dehn twist to construct an example of a geometrically finite branched covering. This example has no Thurston obstruction and is not locally combinatorially attracting at a limiting point of its post-critical set. Thus, it is not combinatorially equivalent to a rational map. (See Theorem 4.1.)

Finally, in $\S 5$, we introduce our combinatorial and locally holomorphical equivalence relation among all sub-hyperbolic semi-rational branched coverings (see Definition [5.1).

\section{Geometric finiteness}

Suppose $S^{2}$ is the two-sphere. We also use $\hat{\mathbb{C}}$ to denote the two-sphere with the standard complex structure. Let $f: S^{2} \rightarrow S^{2}$ be a branched covering of degree $d \geq 2$, which is a continuous orientation-preserving map from the two-sphere onto itself and which is locally homeomorphic except for a finite number of points, and at these finitely many points, which is locally $k$ to 1 for $1<k \leq d$.

By the definition, a point $p$ is called a branched point if $\operatorname{deg}_{x} f>1$. The critical set $\Omega=\Omega_{f}$ of $f$ is the set of all branched points of $f$. The post-critical set $P=P_{f}$ of $f$ is

$$
P=\overline{\bigcup_{n=1}^{\infty} f^{n}(\Omega) .}
$$

The map $f$ is called critically finite if the number $\#(P)<\infty$.

Suppose $f, g: S^{2} \rightarrow S^{2}$ are branched coverings. They are said to be combinatorially equivalent if there is a pair of orientation-preserving homeomorphisms $(\phi, \psi)$ of $S^{2}$ such that the diagram

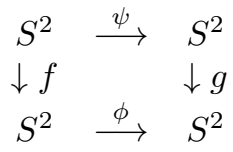

commutes, that is, $\phi f=g \psi$, and $\psi$ is isotopic to $\phi$ rel $P=P_{f}$. Thus, there is a continuous function $F(t, x):[0,1] \times S^{2} \rightarrow S^{2}$ such that for each $0 \leq t \leq 1$, $\phi_{t}(x)=F(t, x)$ is an orientation-preserving homeomorphism of $S^{2}$ and $F(t, p)=$ $\phi(p)=\psi(p)$ for all $p \in P$ and $F(0, x)=\phi(x)$ and $F(1, x)=\psi(x)$ for all $x \in S^{2}$. Here $(\phi, \psi)$ is called a combinatorial equivalence between $f$ and $g$.

Suppose $f: S^{2} \rightarrow S^{2}$ is a branched covering. A simple closed curve in $S^{2}-P$ is called essential if it does not bound a disk in $S^{2}-P$ and peripheral if it encloses a single point of $P$.

A multicurve $\Gamma=\left\{\gamma_{i}\right\}$ on $S^{2}-P$ is a finite non-empty collection of disjoint simple closed curves such that each of the curves is essential and non-peripheral and no two curves are isotopic rel $P$. A multicurve $\Gamma$ is called $f$-invariant if for any $\gamma \in \Gamma$, every non-peripheral essential component of $f^{-1}(\gamma)$ is homotopic to an element of $\Gamma$ rel $P$. 
Suppose $\Gamma$ is a multicurve. Then $\Gamma$ determines a transition matrix $A=A_{\Gamma}=$ $\left(a_{\delta \gamma}\right): \mathbb{R}^{\Gamma} \rightarrow \mathbb{R}^{\Gamma}$ by the formula:

$$
a_{\delta \gamma}=\sum_{\alpha} \frac{1}{\operatorname{deg}(f: \alpha \rightarrow \gamma)},
$$

where the sum is taken over components $\alpha$ of $f^{-1}(\gamma)$ which are homotopic to $\delta$ rel $P$. Since every entry in $A$ is non-negative, the Perron-Frobenius Theorem implies that $A$ has a non-negative and maximum eigenvalue $\lambda=\lambda_{\Gamma}$. An $f$-invariant multicurve $\Gamma$ is called a Thurston obstruction if the maximum eigenvalue $\lambda \geq 1$.

If $f$ is critically finite, for every point $x$ in $S^{2}$, define $v_{f}(x)$ as the least common multiple of local degrees $\operatorname{deg}_{y} f^{n}$ for all $n>0$ and all $y \in S^{2}$ such that $f^{n}(y)=x$.

Note that $v_{f}(x)=1$ if $x$ is not in $P$ and that $v_{f}(x)$ may be $\infty$. Then

$$
\mathcal{O}=\mathcal{O}_{f}=\left(S^{2}, v_{f}\right)
$$

is the orbifold associated to $f$, where

$$
v_{f}: P \rightarrow \mathbb{N} \cup\{\infty\}
$$

is called the signature of $\mathcal{O}$.

A complete proof of the following Thurston's Theorem is given in DouadyHubbard's paper 6 .

Theorem 2.1. Suppose that $f$ is a critically finite branched covering of degree $d \geq 2$. If the signature of $\mathcal{O}$ is not $(2,2,2,2)$, then $f$ is combinatorially equivalent to a rational map $R$ if and only if $f$ has no Thurston obstruction. Moreover, the rational map $R: \hat{\mathbb{C}} \rightarrow \hat{\mathbb{C}}$ is unique up to conjugation by Möbius transformations.

Thurston's condition for $f$ is defined to mean that $f$ has no Thurston obstruction. So the above theorem says that Thurston's condition is necessary and sufficient for a critically finite rational map whose signature of $\mathcal{O}$ is not $(2,2,2,2)$ among all critically finite branched coverings under the combinatorial equivalence relation.

The following McMullen's Theorem is proved in 9.

Theorem 2.2. Suppose $R: \hat{\mathbb{C}} \rightarrow \hat{\mathbb{C}}$ is a rational map. Let $\Gamma$ be a multicurve on $\hat{\mathbb{C}}-P$ where $P=P_{R}$. Then $\lambda \leq 1$. The equality holds only in the following cases:

- $R$ is critically finite and the signature of $\mathcal{O}_{R}$ is $(2,2,2,2)$.

- $P$ is an infinite set, and $\Gamma$ includes the essential curves in a finite system of annuli permuted by $R$. These annuli lie in Siegel disks or Herman rings for $R$, and each annulus is a connected component of $\hat{\mathbb{C}}-P$.

A rational map is called geometrically finite if

$$
\#(P)=\infty \text { but } \#(P \cap J)<\infty,
$$

where $J$ is the Julia set of $R$ (see [10]). We use $P^{\prime}$ to denote the set of all limiting

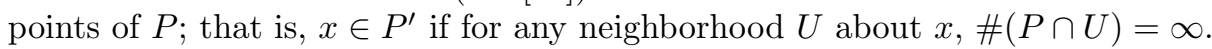

Theorem 2.3. A rational map $R$ is critical finite or geometrically finite if and only if $P^{\prime}$ is finite.

Before we prove this theorem, let us review Mañé's Theorem. For a rational map $R$, a branched point $c$ is also called a critical point. (Note that $R^{\prime}(c)=0$ for 
a critical point $c$.) Let $c$ be a critical point of $R$. The $\omega$-limit set $\omega(c)$ of $c$ is, by definition,

$$
\omega(c)=\left\{z \in \hat{\mathbb{C}} \mid \lim _{k \rightarrow \infty} R^{n_{k}}(c)=z \text { for some subsequence }\left\{n_{k}\right\}_{k=1}^{\infty} \text { of integers }\right\} .
$$

A critical point $c$ is called recurrent if $c \in \omega(c)$. The following Mañe's Theorem is proved in [8].

Theorem 2.4. Suppose $R$ is a rational map and $J$ is its Julia set. Let $\Lambda \subset J$ be a compact forward invariant set, which means, $R(\Lambda) \subseteq \Lambda$. If $\Lambda$ contains neither critical points nor parabolic periodic points and if $\Lambda$ is disjoint from the $\omega$-limit set of every recurrent critical point, then $R$ on $\Lambda$ is expanding. This means that there are two constants $C>0$ and $\lambda>1$ such that

$$
\left|\left(R^{n}\right)^{\prime}(z)\right| \geq C \lambda^{n}, \quad \forall n>0, \forall z \in \Lambda .
$$

Proof of Theorem 2.3. It is known that a critical finite or geometrically finite rational map has no Siegel disk and Herman ring since the boundary of a Siegel disk or Herman ring is contained in the $\omega$-limit set of critical points (refer to [10, 146-151]). Suppose $R$ is critical finite or geometrically finite. Then $P \cap J_{R}$ is finite. Suppose $F$ is the Fatou set of $R$. For every critical point $c \in F, \omega(c)$ is an attractive, superattractive, or parabolic cycle. So $P^{\prime} \cap F$ is finite. Thus $P^{\prime} \subset\left(P^{\prime} \cap F\right) \cup\left(P^{\prime} \cap J\right)$ is finite.

Conversely, if $P^{\prime}$ is finite, then for every $c \in J, \omega(c)$ is finite and hence a periodic cycle. In particular, every critical point on the Julia set is non-recurrent. We claim that every critical point on the Julia set is eventually periodic. This implies that the rational map $R$ is critical finite or geometrically finite.

Now let us prove the claim. Suppose a critical point $c \in J$ is not eventually periodic. Consider the periodic cycle $\omega(c)$. If it is parabolic, from Fatou's Flower Theorem (see [10]), $R^{n}(c)$ must tend to this cycle from attracting petals. This is impossible since $R^{n}(c) \in J$. So it cannot be parabolic. From Mañé's Theorem, this periodic cycle is repelling. This is also impossible. So every critical point in the Julia set $J$ is eventually periodic. We have proved the theorem.

Following Theorem 2.3, we now give our definition of a geometrically finite branched covering, which is compatible with the traditional definition of a geometrically finite rational map.

Definition 2.1. A branched covering $f$ of the two-sphere of degree $d \geq 2$ is called geometrically finite if its post-critical set $P$ contains infinitely many points but the limiting set $P^{\prime}$ of its post-critical set contains only finitely many points.

For a geometrically finite branched covering $f$, each point $x \in P^{\prime}$ is periodic. Thus $P^{\prime}$ consists of a finite number of periodic cycles. (This is an easy exercise. We leave it for the reader.)

\section{Local COMBinatorial ATtractive}

We first give the following definitions:

Definition 3.1. Suppose $f: \hat{\mathbb{C}} \rightarrow \hat{\mathbb{C}}$ is a geometrically finite branched covering of degree $d \geq 2$. We say $f$ is semi-rational if

(1) $f$ is holomorphic in a neighborhood of $P^{\prime}$; 
(2) each cycle $\left\langle p_{0}, \cdots, p_{k-1}\right\rangle$ of period $k \geq 1$ in $P^{\prime}$ is either attractive, that is, $0<\left|\left(f^{k}\right)^{\prime}\left(p_{0}\right)\right|<1$, or super-attractive, that is, $\left(f^{k}\right)^{\prime}\left(p_{0}\right)=0$, or parabolic, that is, $\left|\left(f^{k}\right)^{\prime}\left(p_{0}\right)\right|=1$ and $\left(\left(f^{k}\right)^{\prime}\left(p_{0}\right)\right)^{q}=1$ for some integer $q \geq 1$; and

(3) each attracting petal associated with a parabolic cycle in $P^{\prime}$ contains a point in the post-critical set $P$.

Furthermore, if all cycles in $P^{\prime}$ are either attractive or super-attractive, we call $f$ a sub-hyperbolic semi-rational branched covering. Clearly, every geometrically finite rational map is a semi-rational branched covering.

See 10 for the terminology of an attracting petal associated with a parabolic cycle in this definition.

Definition 3.2. Suppose $f: \hat{\mathbb{C}} \rightarrow \hat{\mathbb{C}}$ is a geometrically finite branched covering of degree $d \geq 2$. Suppose that $p \in P^{\prime}$ is a periodic point of period $k \geq 1$. We say $f$ is locally combinatorially attracting at $p$ if there exists a combinatorial equivalence $(\phi, \psi)$ from $f$ to a branched covering $g: \hat{\mathbb{C}} \rightarrow \hat{\mathbb{C}}$ such that $g$ is holomorphic in a neighborhood of the forward orbit of $\phi(p)$ under $g$ and such that $\phi(p)$ is a holomorphically attracting or superattracting fixed point of $g^{k}$, that is, $0 \leq\left|\left(g^{k}\right)^{\prime}(\phi(p))\right|<1$.

From the above definitions, it is easy to verify that if a geometrically finite branched covering $f$ is locally combinatorially attracting at every point of $P^{\prime}$, then it is combinatorially equivalent to a sub-hyperbolic semi-rational branched covering $g$. One of the interesting results in this paper is that

Theorem 3.1. A semi-rational branched covering $f$ is always combinatorially equivalent to a sub-hyperbolic semi-rational branched covering $g$.

Before proving Theorem 3.1, we first study some local combinatorial properties around a point in $P^{\prime}$. Suppose $f$ is a geometrically finite branched covering.

Definition 3.3. Suppose $p \in P^{\prime}$ is a periodic point of period $k \geq 1$. Suppose $\Gamma=\left\{\gamma_{n}\right\}_{n=0}^{\infty}$ is a family of disjoint pairwise simple closed curves in $\hat{\mathbb{C}}-P$. We call $\Gamma$ a combinatorially invariant family of curves nested at $p$ if for all $n \geq 0, \gamma_{n+1}$ separates $\gamma_{n}$ from $p$ and there is a component of $f^{-k}\left(\gamma_{n+1}\right)$ homotopic to $\gamma_{n}$ rel $P$.

Definition 3.4. A combinatorially invariant family of curves $\Gamma$ nested at $p \in P^{\prime}$ is called shrinking if for every neighborhood $U$ of $p$, there is a simple closed curve $\beta \subset U-P$ such that $\beta$ is homotopic to some $\gamma_{n} \in \Gamma$ rel $P$.

The next lemma says that Definition 3.2 and Definition 3.4 are essentially the same at a point $p \in P^{\prime}$ which is not a branched point of $f$.

Lemma 3.1. Suppose $p \in P^{\prime}$ is a periodic point of period $k \geq 1$ and $\operatorname{deg}_{p} f^{k}=1$. Then $f$ is locally combinatorially attracting at $p$ if and only if there is a combinatorially invariant shrinking family of curves nested at $p$.

Proof. Suppose $f$ is locally combinatorially attracting at $p$, i.e., suppose there exists a combinatorial equivalence $(\phi, \psi)$ from $f$ to a branched covering $g: \hat{\mathbb{C}} \rightarrow \hat{\mathbb{C}}$ such that $\phi(p)$ is a holomorphic attracting fixed point of $g^{k}$. By Koenig's Theorem (refer to [10]), we may suppose that $\phi(p)=0$ and $g^{k}(z)=\lambda z$ in a neighborhood $U$ of the origin for some constant $\lambda$ with $0<|\lambda|<1$.

Since $g$ has only finitely many critical points, there exists a disk $D=\left\{z|| z \mid<r_{0}\right\}$ in $U$ such that for each critical point $c$ of $g$, either its forward orbit never lands 
in $U-\{0\}$ or the first point in $P$ hitting $U-\{0\}$ lands in $U-D$. Pick a circle $C=\left\{z|| z \mid=r_{1}\right\}$ in $D$ such that $C$ is disjoint from $P_{g}$. Then $g^{n p}(C)$ is also disjoint from $P_{g}$ for all $n \geq 1$. Let $\gamma_{n}=\phi^{-1}\left(g^{n k}(C)\right)$. It is clear that $\Gamma=\left\{\gamma_{n}\right\}_{n=0}^{\infty}$ is a combinatorially invariant shrinking family of curves nested at $p$.

Conversely, suppose $\Gamma=\left\{\gamma_{n}\right\}_{n=0}^{\infty}$ is a combinatorially invariant shrinking family of curves nested at $p$. Without loss of generality, we assume that $\gamma_{n}$ converges to $p$ in the spherical distance as $n$ goes to infinity in the spherical distance.

Since $\operatorname{deg}_{p} f^{k}=1$, there exists a simply connected neighborhood $U$ of $p$ such that the restriction of $f^{k}$ on $U$, denoted by $F$, is a homeomorphism. There exists an integer $n_{0} \geq 0$ such that for $n \geq n_{0}, \gamma_{n+1}$ is contained in $F(U)$ and $\beta_{n}=F^{-1}\left(\gamma_{n+1}\right)$ is homotopic to $\gamma_{n}$ rel $P_{f}$. Clearly, $\beta_{n}$ converges to $p$ as $n$ goes to infinity in the spherical distance.

Both $\gamma_{n}$ and $\beta_{n}$ converge to $p$ as $n$ goes to infinity in the spherical distance. Since $\gamma_{n}$ and $\beta_{n}$ are simple closed curves, then there exists an orientation-preserving homeomorphism $\theta$ of $\hat{\mathbb{C}}$ such that $\theta$ is isotopic to the identity rel $P$ and $\theta\left(\gamma_{n}\right)=\beta_{n}$ for $n \geq n_{0}$. Moreover, the map $\theta$ can be chosen such that it is the identity in a neighborhood of $\left\{f(p), \cdots, f^{k-1}(p)\right\}$. This implies that

$$
f^{k} \circ \theta\left(\gamma_{n}\right)=(f \circ \theta)^{k}\left(\gamma_{n}\right)=\gamma_{n+1}
$$

for $n \geq n_{1}$, where $n_{1}>n_{0}$ is a constant.

Since $\gamma_{n}$ converges to $p$ in the spherical distance as $n$ goes to infinity, there exists an orientation-preserving homeomorphism $\phi: \hat{\mathbb{C}} \rightarrow \widehat{\mathbb{C}}$ such that $\phi\left(\gamma_{n}\right)$ is the circle $\left\{z:|z|=\lambda^{n} r\right\}$ for $n \geq n_{1}$, where $\lambda$ and $r$ are positive constants with $\lambda<1$. Moreover, the map $\phi$ can be chosen such that the restriction of $\phi$ on $\gamma_{n_{1}+k}$ is equal to $g \phi F^{-l}$ for every integer $l>0$, where $g(z)=\lambda z$. Furthermore, the map $\phi$ can also be chosen such that the restriction of $\phi$ on the ring bounded by $\gamma_{n_{1}+l}$ and $\gamma_{n_{1}+l+1}$ is equal to $g \phi F^{-l}$ for every integer $l>0$.

Now $\phi f^{k} \theta \phi^{-1}(z)=\left(\phi f \theta \phi^{-1}\right)^{k}=g(z)$ on a neighborhood of the origin. This means that $f$ is locally combinatorially attracting at $p$. We have proved the lemma.

Proof of Theorem 3.1. Suppose that $p \in P^{\prime}$ is a parabolic periodic point of period $k \geq 1$. Let $V$ be an attracting flower of $f^{k}$ associated with $p$ (refer to the LeauFatou Flower Theorem in [10]). Let $\gamma_{0} \subset \widehat{\mathbb{C}}-P$ be a simple closed curve such that one component $U_{0}$ of $\hat{\mathbb{C}}-\gamma_{0}$ contains $\overline{f^{k}(V)}$ and $U_{0} \cap P=\overline{f^{k}(V)} \cap P$. Inductively, for all $n \geq 1$, let $\gamma_{n} \subset U_{n-1}-P$ be a simple closed curve such that one component $U_{n}$ of $\hat{\mathbb{C}}-\gamma_{n}$ contains $\overline{f^{(n+1) k}(V)}$ and $U_{n} \cap P=\overline{f^{n k}(V)} \cap P$. Then $\left\{\gamma_{n}\right\}_{n=0}^{\infty}$ is a combinatorially invariant shrinking family of curves nested at $p$. So $f$ is locally combinatorially attracting at $p$ by Lemma 3.1. Since $p \in P^{\prime}$ is any parabolic periodic point, then $f$ is combinatorially equivalent to a sub-hyperbolic semi-rational branched covering $g$. This completes the proof.

\section{ThuRSton's CONDITION IN THE GEOMETRICALlY FINITE CASE}

In this section we prove that Thurston's condition is not necessary for a geometrically finite rational map among all geometrically finite branched coverings under the combinatorial equivalence relation. Suppose $f$ is a geometrically finite branched covering of degree $d \geq 2$. 
Lemma 4.1. Suppose $p \in P^{\prime}$ is a fixed point of $f$ with $\operatorname{deg}_{p} f=1$. If $f$ is locally combinatorially attracting at $p$, then there is a neighborhood $V$ of $p$ such that any combinatorially invariant family of curves nested at $p$ in $V$ is shrinking.

Proof. By the definition and Koenig's Theorem (see [10]), there exists a combinatorial equivalence $(\phi, \psi)$ from $f$ to a branched covering $g: \hat{\mathbb{C}} \rightarrow \hat{\mathbb{C}}$ such that $\phi(p)=0$ and $g(z)=\lambda z$ on a neighborhood $U$ of 0 for some constant $\lambda$ with $0<|\lambda|<1$.

Since $g$ has only finitely many critical points, there exists a constant $r>0$ such that $D(r)=\{z \in \mathbb{C}|| z \mid<r\} \subset U$ satisfies the statement that for all integers $n \geq 0$,

$$
P_{g} \cap \partial D\left(|\lambda|^{n} r\right)=\emptyset \quad \text { and } \quad P_{g} \cap A_{n}=g^{n}\left(P_{g} \cap A_{0}\right),
$$

where

$$
A_{n}=\left\{\left.z \in \mathbb{C}|| \lambda\right|^{n+1} r<|z|<|\lambda|^{n} r\right\} .
$$

For any simple closed curves $\alpha_{1}$ and $\alpha_{2}$ in $D(r)-P_{g}$, if $\alpha_{1}$ is isotopic to $\alpha_{2}$ rel $P_{g}$, then $g\left(\alpha_{1}\right)$ is isotopic to $g\left(\alpha_{2}\right)$ rel $P_{g}$.

Let $V=\phi^{-1}(D(|\lambda| r))$. Suppose that $\Gamma=\left\{\gamma_{n}\right\}_{n=0}^{\infty}$ is a combinatorially invariant family of curves nested at $p$ in $V$. Let $\beta_{n}=\phi\left(\gamma_{n}\right)$. Then $\beta_{n}=\phi\left(\gamma_{n}\right) \subset D(|\lambda| r)$ and $g^{-1}\left(\beta_{n+1}\right) \cap D(r)$ is isotopic to $\beta_{n}$ rel $P_{g}$. Thus $\beta_{n+1}$ is isotopic to $g\left(\beta_{n}\right)$ rel $P_{g}$. Hence it is also isotopic to $g^{n}\left(\beta_{1}\right)$ rel $P_{g}$ for all $n \geq 1$. We know that $g^{n}\left(\beta_{1}\right)$ converges to the origin in the spherical distance as $n \rightarrow \infty$, but $\gamma_{n+1}$ is isotopic to $\phi^{-1} g^{n}\left(\beta_{1}\right)$ rel $P$. So $\Gamma_{n}$ is shrinking. We have proved the lemma.

Lemma 4.2. Suppose $p \in P^{\prime}$ is a fixed point with $\operatorname{deg}_{p} f=1$. If there is only one critical point whose forward orbit converges to $p$ in the spherical distance, then there is at most one combinatorially invariant shrinking family of curves nested at $p$ up to isotopy rel $P$. This means that if $\Gamma=\left\{\gamma_{n}\right\}_{n=0}^{\infty}$ and $\Gamma^{\prime}=\left\{\beta_{n}\right\}_{n=0}^{\infty}$ are combinatorially invariant shrinking families of curves nested at $p$, then there exist integers $k$ and $m \geq 1$ such that $\gamma_{n+k}$ is isotopic to $\beta_{n}$ rel $P$ for all $n \geq m$.

Proof. Suppose that $f$ has a combinatorially invariant shrinking family of curves nested at $p$. By Lemma 3.1, $f$ is locally combinatorially attracting at $p$, which means that there exists a combinatorial equivalence $(\phi, \psi)$ from $f$ to a branched covering $g: \hat{\mathbb{C}} \rightarrow \hat{\mathbb{C}}$ such that $\phi(p)=0$ and $g(z)=\lambda z(0<|\lambda|<1)$ on a neighborhood $U$ of 0 (refer to the proof of Lemma 3.1).

Consider the quotient space $T=U /\langle g\rangle$. It is a torus with a marked point corresponding to the unique critical orbit attracting to 0. Each invariant shrinking family of curves about 0 projects to a homotopy class of $(1,0)$-type simple closed curves on $T$. Since there is only one marked point on $T$, the homotopy class of $(1,0)$ type simple closed curves on $T$ is unique. Thus there is only one invariant shrinking family of curves about 0 up to isotopy rel $P_{g}$. So there is only one combinatorially invariant shrinking family of curves nested at $p$ up to isotopy rel $P$. This completes the proof of the lemma.

Concluding from our two lemmas above, we now can construct an example of a geometrically finite branched covering. This example satisfies Thurston's condition (that is, it has no Thurston obstruction) but is not combinatorially equivalent to any rational map.

Theorem 4.1. There exists a geometrically finite branched covering $f$ such that $f$ has no Thurston obstruction and such that $f$ is not combinatorially equivalent to any rational map. 
Proof. Consider the quadratic polynomial $q(z)=\lambda z+z^{2}, 0<|\lambda|<1$. The point $c=-\lambda / 2$ is the unique critical point of $q$ in the complex plane $\mathbb{C}$. Let $P O=\left\{c_{n}=q^{n}(c)\right\}_{n=1}^{\infty}$ be the orbit of $q(c)$. Then it converges to 0 as $n \rightarrow \infty$ in the spherical distance, which is the unique attractive fixed point of $q$ in $\mathbb{C}$. Note that $P_{q}=P O \cup\{0, \infty\}$.

There is a combinatorially invariant shrinking family of curves $\Gamma=\left\{\alpha_{n}\right\}_{n=0}^{\infty}$ nested at 0 such that for $U_{n}$, the domain bounded by $\alpha_{n}$, we have that $q \mid U_{0}$ is univalent, $U_{0} \supset P$, and $U_{n}=q^{n}\left(U_{0}\right)$. There is an $\operatorname{arc} \beta$ in $U_{0}$ which connects $c_{1}$ with 0 and passes over every point $c_{n}$ such that $q(\beta) \subset \beta$ and $\beta$ intersects with each $\alpha_{n}(n>0)$ at one point (see Figure 1).

Denote by $A$ the annulus bounded by $\alpha_{0}$ and $\alpha_{2}$. Take $\delta$ to be a Jordan curve in $A$ such that $\delta$ intersects with $\beta$ at one point and $\delta$ bounds a disk $D$ in $A$ containing $c_{1}$ and $c_{2}$ (see Figure 1).

Let $T_{0}$ be the Dehn twist along $\delta$ (see Figure 2). Here $T_{0}$ can be picked as a homeomorphism which is the identity out of an annulus neighborhood $C(\delta)$ of $\delta$ in $A-\left\{c_{1}, c_{2}\right\}$ and which is $h^{-1} \circ R \circ h$ on $C(\delta)$, where $h$ is a conformal map from $C(\delta)$ to a standard annulus $C=\{z|0<r<| z \mid<1\}$ and $R$ is a homeomorphism from $C$ to itself defined as

$$
R\left(\rho e^{i \theta}\right)=\rho e^{i\left(\theta+2 \pi \frac{\rho-r}{1-r}\right)}, \quad r<\rho<1 .
$$

Define $T_{2 n}=q^{2 n} T_{0} q^{-2 n}$ on $q^{2 n}(C(\delta))$ and the identity elsewhere. Let

$$
f_{n}=q \circ T_{0}^{2} \circ T_{2}^{2} \circ \cdots \circ T_{2 n}^{2} .
$$

For any $x \neq 0$, there is an $n(x) \geq 1$ such that $f_{n}(x)=f_{n(x)}(x)$ for all $n \geq n(x)$. Define $f(x)=f_{n}(x)$ for all $n \geq n(x)$. Since $f_{n}(0)=0$ for all $n \geq 1$, define $f(0)=0$. For any $\epsilon>0$, there is a $\delta>0$ such that $f_{n}\left(\Delta_{\delta}\right) \subset \Delta_{\epsilon}$ for all $n$ large, where

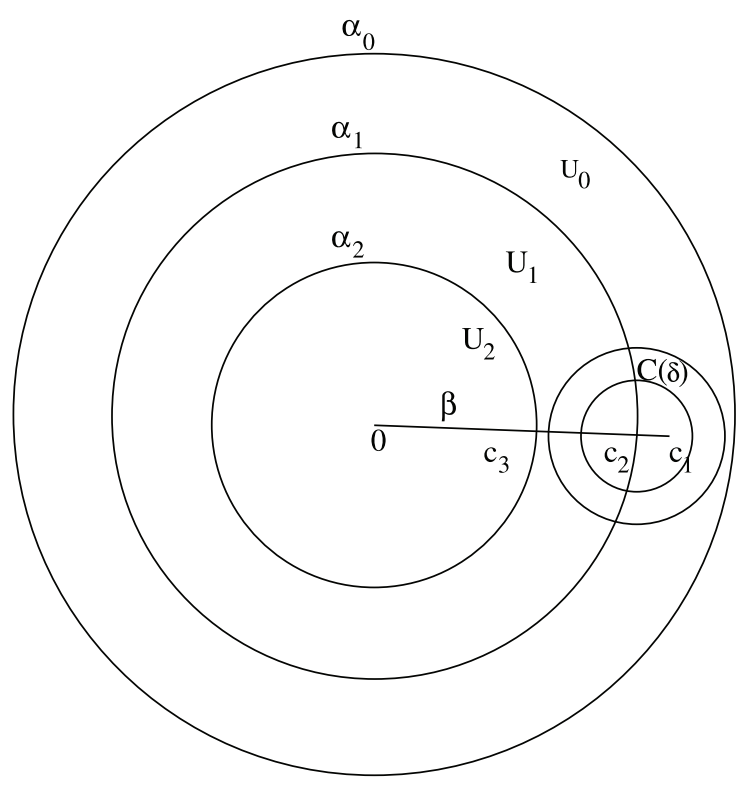

Figure 1 
$\Delta_{\delta}=\{z \in \mathbb{C}|| z \mid<\delta\}$ and $\Delta_{\epsilon}=\{z \in \mathbb{C}|| z \mid<\epsilon\}$. So $f\left(\Delta_{\delta}\right) \subset \Delta_{\epsilon}$. Thus $f$ is continuous at 0 . If we consider the local inverse branch of $f_{n}^{-1}$ fixing 0 , similarly, we can define $f^{-1}$ locally at 0 and fixing 0 , such that $f^{-1}$ is continuous at 0 . Thus $f$ is locally homeomorphic at 0 . Thus $\operatorname{deg}_{0} f=1$, and $f: \hat{\mathbb{C}} \rightarrow \hat{\mathbb{C}}$ is a branched covering of degree 2. The sequence $\left\{f_{n}\right\}$ of branched coverings converges to $f$ as $n$ goes to $\infty$ in the maximal norm with respect to the spherical distance. In particular,

$$
P=P_{q}=P O \cup\{0, \infty\}
$$

(see Figure 2).

Let $\gamma_{n}^{n}=\alpha_{2 n}$ and $\gamma_{n+k}^{n}=f^{k}\left(\gamma_{n}^{n}\right) \subset U_{2 n}$ for $k>0$. Since $\gamma_{n+1}^{n}=f\left(\gamma_{n}^{n}\right)=\alpha_{2 n+1}$, we see that $\Gamma^{n}=\left\{\gamma_{n+k}^{n}\right\}_{k=1}^{\infty}$ is a combinatorially invariant family of curves nested at 0 for all $n \geq 0$.

Since $f\left(\gamma_{n+1}^{n}\right)$ is not isotopic to $\gamma_{n+1}^{n+1}$ rel $P$ (refer to Figure 2), each curve in $\Gamma^{n}$ is not isotopic to any curve in $\Gamma^{n+1}$. If $\Gamma^{m}$ is shrinking for some $m$, so is $\Gamma^{m+1}$ since $\gamma_{m+1}^{m+1}$ separates $\gamma_{m}^{m}$ from 0 . Thus $\Gamma^{n}$ is not shrinking for all $n \geq 0$ by Lemma 4.2. Because $\gamma_{n}^{n}$ converges to 0 in the spherical distance as $n$ goes to $\infty, f$ has no combinatorially invariant shrinking family of curves nested at 0 and hence is not locally combinatorially attracting at 0 by Lemma 3.1 and Lemma 4.1 This proves that $f$ cannot be combinatorially equivalent to a rational map.

Now we show that $f$ has no Thurston obstruction. For every $n \geq 0$, let $f^{-n}$ be the maximal local inverse branch of $f^{n}$ fixing 0 . We prove by induction that $f^{-2 n}\left(U_{2 n}\right) \supset P$ for all $n \geq 0$.

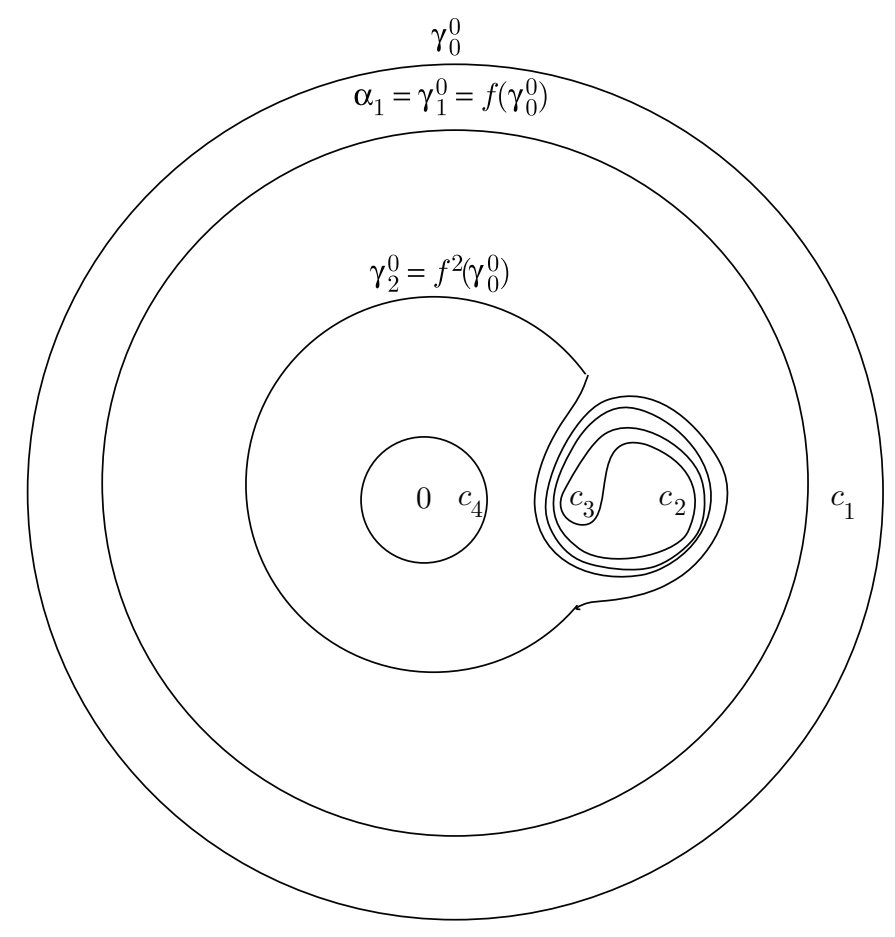

Figure 2 
Clearly $U_{0} \supset P$. Assume $f^{-2 n+2}\left(U_{2 n-2}\right) \supset P$ for $n \geq 1$. Note that

$$
U_{2 n-1} \supset U_{2 n} \supset\left\{c_{2 n+1}, c_{2 n+2}, \cdots\right\} .
$$

Since $f^{-2 n+1}\left(U_{2 n-1}\right)=f^{-2 n+2}\left(U_{2 n-2}\right) \supset P$ and $f^{2 n-1}$ is a homeomorphism on $f^{-2 n+1}\left(U_{2 n-1}\right)$, we have $f^{-2 n+1}\left(U_{2 n}\right) \supset P-\left\{c_{1}\right\}$. Let $\beta_{n}$ be the segment of $\beta$ with endpoints $c_{n}$ and $c_{n+1}$ for $n \geq 1$. Then $\beta_{n+1} \subset U_{n}$ for $n \geq 0$. In particular, $\beta_{2 n}$ connects $c_{2 n}$ and $c_{2 n+1}$ in $U_{2 n-1}$, and $\beta_{2 n+1}$ connects $c_{2 n+1}$ with $c_{2 n+2}$ in $U_{2 n}$. By the definition of $f, f^{-1}\left(\beta_{2 n+1}\right)=T_{2(n-1)}^{-2}\left(\beta_{2 n}\right)$ (refer to Figure 3). So $\beta_{2 n} \cup f^{-1}\left(\beta_{2 n+1}\right)$ is a loop whose winding number with respect to $c_{2 n-1}$ is \pm 2 .

Now, $f^{-2 n+2}\left(\beta_{2 n}\right)$ connects $c_{2}$ with $c_{3}$ in $f^{-2 n+2}\left(U_{2 n-1}\right)$, and $f^{-2 n+1}\left(\beta_{2 n+1}\right)$ connects $c_{2}$ with $c_{3}$ in $f^{-2 n+1}\left(U_{2 n}\right)$. Their union is also a loop whose winding number with respect to $c_{1}$ is also \pm 2 (see Figure 3).

By the assumption $f^{-2 n+2}\left(U_{2 n-2}\right) \supset P$, we know that $f^{-2 n+1}\left(\beta_{2 n}\right)$ connects $c_{2}$ with $c_{1}$. Since $c_{1}$ is a critical value of degree 2 , the path $f^{-2 n}\left(\beta_{2 n+1}\right)$ must connect $c_{2}$ with $c_{1}$ in $f^{-2 n}\left(U_{2 n}\right)$. This means that

$$
f^{-2 n}\left(U_{2 n}\right) \supset P
$$

(see Figure 3) .

Suppose that $\Gamma$ is an $f$-invariant multicurve. Denote by $A=A_{\Gamma}$ the transition matrix determined by $\Gamma$. For any element $\tau \in \Gamma$, since $\gamma_{n}^{n}$ converges to zero in the spherical distance as $n$ goes to $\infty$, there is $m>0$ such that $\tau$ is outside the closure of $U_{2 m}$. Since $f^{-2 m}\left(U_{2 m}\right) \supset P$ is a Jordan domain, each component of the preimage of $\tau$ under $f^{-2 m}$ is either non-essential or peripheral. This shows that $A^{2 m}$ is the zero matrix. Hence its spectral radius is 0 . This implies that the spectral radius of $A$ is less than one. So $\Gamma$ is not a Thurston obstruction. Therefore, $f$ satisfies Thurston's condition. This completes the proof.

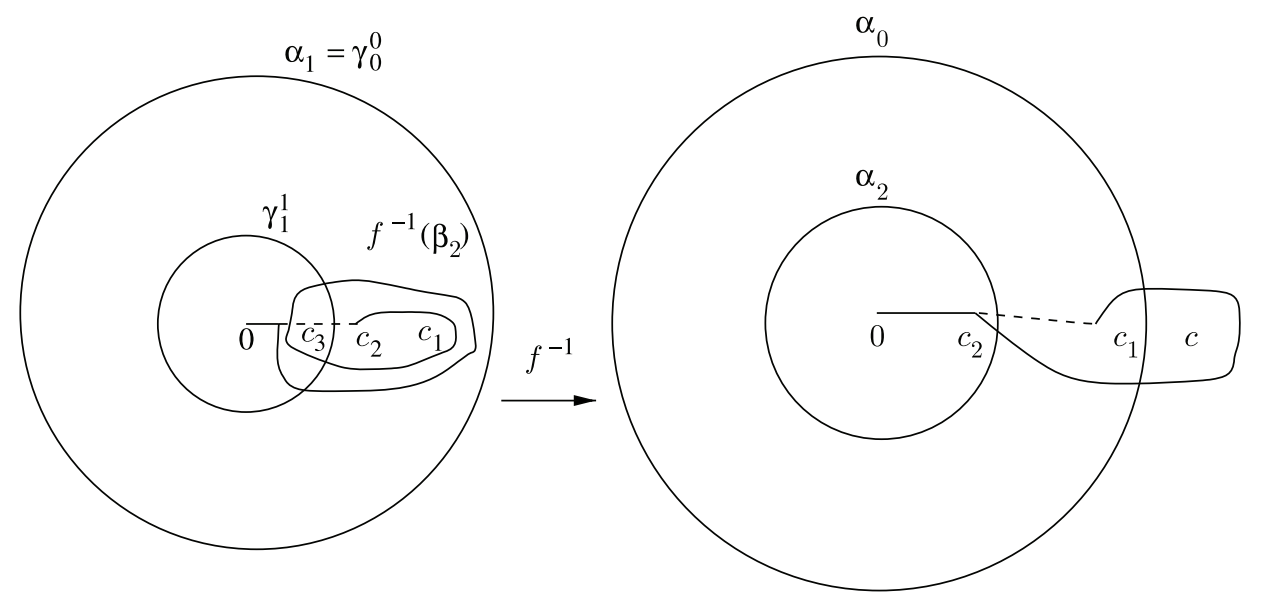

Figure 3 


\section{Combinatorial AND LOCALly hOLOMORPHiCAL EQUIVALENCE RELATION}

Now we introduce our combinatorial and locally holomorphical equivalence relation among all sub-hyperbolic semi-rational branched coverings.

Definition 5.1. Suppose $f$ and $g$ are two sub-hyperbolic semi-rational branched coverings. We say that they are CLH-equivalent (combinatorially and locally holomorphically equivalent) if there exist a pair of orientation-preserving homeomorphisms $\phi: \widehat{\mathbb{C}} \rightarrow \widehat{\mathbb{C}}$ and $\psi: \widehat{\mathbb{C}} \rightarrow \widehat{\mathbb{C}}$ such that

1) $\psi$ is isotopic to $\phi$ rel $P_{f}$,

2) $\phi f=g \psi$,

3) $\phi\left|U_{f}=\psi\right| U_{f}$ is holomorphic on some open set $U_{f} \supset P_{f}^{\prime}$.

Under this CLH-equivalence relation, we have stated Theorem 1.1] in [4. The idea in our proof in [4] is to apply some surgery method and to reduce the problem to that of Thurston's original postcritically finite setup (see Theorem 2.1). In this proof, we need to check that certain gluing data are analytically realizable. Therefore, the proof is quite involved. In the paper [12], a new and simpler proof is given by completely giving up the idea in the old proof. This new proof adapted some arguments used in the proof in Douady and Hubbard's paper [6] and also used the idea of constructing a shielding ring around every point in the limiting set of the post-critical set. Then we proved that the bounded geometry is valid due to these shielding rings. Thus this proof is complete and understandable and checkable. Finally, by combining Theorem 1.1 and Theorem 3.1, we have Theorem 1.2, which is one of the main results in this paper.

\section{ACKNOWLEDGMEnTs}

The authors would like to thank the referee for his careful reading of the original version of this paper and for many extremely useful suggestions and comments. The authors were partially supported by grants from NSF, PSC-CUNY, NSFC (grant \#10831004) and BaiRenJiHua.

\section{REFERENCES}

[1] D. Brown, Spider theory to explore parameter spaces. Cornell University Ph.D. thesis, 2001. Stony Brook thesis preprint server.

[2] Guizhen Cui, Yunping Jiang, and Dennis Sullivan, Dynamics of geometrically finite rational maps. Manuscript, 1994.

[3] G. Cui, Y. Jiang, and D. Sullivan, On geometrically finite branched coverings. I. Locally combinatorial attracting. Complex Dynamics and Related Topics, New Studies in Advanced Mathematics, Vol. 5, 2004, The International Press, 1-14. MR.2504307

[4] G. Cui, Y. Jiang, and D. Sullivan, On geometrically finite branched coverings. II. Realization of rational maps. Complex Dynamics and Related Topics, New Studies in Advanced Mathematics, Vol. 5, 2004, The International Press, 15-29. MR.2504308

[5] G. Cui and L. Tan, A characterization of hyperbolic rational maps. Preprint.

[6] A. Douady and J. H. Hubbard, A proof of Thurston's topological characterization of rational functions. Acta Math., Vol. 171, 1993, 263-297. MR1251582 (94j:58143)

[7] J. H. Hubbard, D. Schleicher, and M. Shishikura, Exponential Thurston maps and limits of quadratic differentials. Journal of Amer. Math. Soc. 22 (2009), 77-117. MR2449055

[8] R. Mañé, On a theorem of Fatou. Bol. Soc. Brasil. Mat. Vol. 24, no. 1 (1993), 1-11. MR:1224298(94g:58188) 
[9] C. McMullen, Complex dynamics and Renormalization. Ann. of Math. Studies, 79, 1994. MR:1312365 (96b:58097)

[10] J. Milnor, Dynamics in One Complex Variable, Introductory Lectures. Vieweg, 2nd Edition, 2000. MR.1721240 (2002i:37057)

[11] G. Zhang, Topological models of polynomials of simple Siegel disk type, CUNY Graduate Center Ph.D. thesis, 2002.

[12] G. Zhang and Y. Jiang, Combinatorial characterization of sub-hyperbolic rational maps. Advances in Mathematics, 221 (2009), 1990-2018. MR2522834

Academy of Mathematics and Systems Science, Chinese Academy of Sciences, Beijing 100190, People's Republic of China

E-mail address: gzcui@math.ac.cn

Department of Mathematics, Queens College of CUny, Flushing, New York 11367 - and - Department of Mathematics, CUNy Graduate Center, New York, New YORK 10016

E-mail address: Yunping.Jiang@qc.cuny.edu 